\title{
EFFECTS OF SOME POTASSIUM PRESERVATIVES ON PHYSIOLOGICAL ACTIVITIES OF SELECTED FOOD BORNE BACTERIA
}

\author{
K.G. Mladenovića*, M.Ž. Muruzovića, O.D. Stefanovića ${ }^{a}$ T.D. Žugić Petrovićb \\ and LJ.R. ČOMIĆa \\ ${ }^{a}$ Department of Biology and Ecology, Faculty of Science, University of Kragujevac, Radoja Domanovića 12, \\ 34000 Kragujevac. Republic of Serbia \\ ${ }^{\mathrm{b}}$ College of Agriculture and Food Technology, Ćirila and Metodija 1, 18400 Prokuplje. Republic of Serbia
}

(Received: 1 June 2017; accepted: 10 August 2017)

\begin{abstract}
The antibacterial activity of potassium metabisulphite, potassium benzoate, potassium propionate, and potassium nitrate were evaluated against 15 species of bacteria using diffusion and microdilution methods. Potassium metabisulphite showed the greatest activity (MIC varied in the range of $0.78 \mathrm{mg} \mathrm{m}^{-1}$ to $3.12 \mathrm{mg} \mathrm{ml}^{-1}$ ), then potassium benzoate $\left(6.25 \mathrm{mg} \mathrm{m}^{-1}\right.$ to $\left.12.5 \mathrm{mg} \mathrm{ml}^{-1}\right)$ followed by potassium propionate and potassium nitrate $(6.25$ $\mathrm{mg} \mathrm{ml}^{-1}$ to $100 \mathrm{mg} \mathrm{ml}^{-1}$ ). Effects of potassium benzoate, potassium propionate, and potassium nitrate on the sugar fermentation, the effect of potassium benzoate on cell membrane permeability and on amylolytic activity of bacteria were tested. The results indicated inhibition of fermentation, loss of intracellular macromolecules (proteins) from treated cells, and inhibition of amylolytic activity.

Keywords: preservatives, fermentation, cell membranes, amylolytic activity, food spoilage bacteria
\end{abstract}

Food contains nutrients, which are necessary for growth and development of beneficial and food borne microorganisms (BAKOČEVIĆ, 2011). Preservatives are compounds that prolong or, under optimum conditions, prevent microbial spoilage of food. They manifest microbiostatic or microbiocidal effects. The mechanisms of antimicrobial activity of preservatives are different depending on the chemical used: interaction with deoxyribonucleic acid (DNA), inhibition of protein synthesis, inhibition of enzymatic activity, cell membrane damage, and decreasing the intracellular $\mathrm{pH}$.

Most of the preservatives that are used presently are more efficient against yeasts and moulds than bacteria (NIKAIDO \& VAARA, 1985). Also, it was confirmed that the mechanism of activity was more complex in Gram-negative than in Gram-positive bacteria (RUSSELL \& GOULD, 1988; BAKOČEVIĆ, 2011). The antimicrobial effects of benzoic and propionic acids in dissociated and undissociated forms were examined. Undissociated forms of preservatives presented stronger antimicrobial effects. After passing through cell membrane, the preservatives dissociated into positive and negative ions exhibiting lower activity (EKLUND, $1985 \mathrm{a}, \mathrm{b})$. The inhibitory effect of potassium metabisulphite, sodium benzoate, and potassium sorbate was examined on different strains of bacteria. Potassium metabisulphite showed the greatest antimicrobial activity (OLADAPO et al., 2014). Preservatives could be used in combination with other food preservation methods to protect food from food borne microorganisms (CAMPOs et al., 2011).

\footnotetext{
* To whom correspondence should be addressed.

Phone: +38 134335 040; fax: +38 134336 223; e-mail: katarinamladenovic90@gmail.com
} 
The aims of this paper were in vitro determination of the minimum inhibitory concentrations of potassium metabisulphite, potassium benzoate, potassium propionate, and potassium nitrate in relation to selected species of bacteria, and examination of the effects of preservatives on the bacterial fermentative ability as well as the effect of potassium benzoate on the cell membrane permeability and on the amylolytic activity of bacteria.

\section{Materials and methods}

\subsection{Determination of antibacterial activity}

Antibacterial activity of preservatives was tested against 15 strains of bacteria (probiotic strains: Lactobacillus plantarum, Bifidobacterium animalis subsp. lactis, and Bacillus subtilis IP 5832), standard strains (Escherichia coli ATCC 25922, Klebsiella pneumoniae ATCC 70063, and Bacillus subtilis ATCC 6633), and clinical isolates (Staphylococcus sp., Escherichia coli, Salmonella enterica, Salmonella typhimurium, Klebsiella pneumoniae, Proteus mirabilis, Pseudomonas aeruginosa, Bacillus cereus, and Bacillus subtilis). All clinical isolates were a generous gift from the Institute of Public Health (Kragujevac, Serbia). Probiotics and ATCC strains were obtained from a collection of the Microbiology Laboratory, Faculty of Science, University of Kragujevac. Bacterial suspensions were prepared by the direct colony method (ANDREws, 2005). The turbidity of initial bacterial suspension (0.5 McFarland) was adjusted using McFarland densitometer (BioSan, Latvia). Initial bacterial suspensions contained about $10^{8}$ colony forming units $(\mathrm{CFU}) \mathrm{ml}^{-1}$. From the initial suspension 1:100 dilutions were additionally prepared in sterile $0.85 \%$ saline solution.

Antibacterial activity was tested by determining the minimum inhibitory concentration (MIC) using microdilution method (SARKER et al., 2007). The stock concentrations of tested preservatives (potassium metabisulphite (Acros Organics, USA), potassium benzoate (Alfa Aesar GmbH \& Co., Germany), potassium propionate (Tokyo Chemical Industry Co., Japan), and potassium nitrate (Alkaloid, Skoplje, Macedonia)) dissolved in liquid nutrient medium were $200 \mathrm{mg} \mathrm{ml}^{-1}$. Next, serial twofold dilutions of each compound were made in a concentration range from $0.04 \mathrm{mg} \mathrm{ml}^{-1}$ to $100 \mathrm{mg} \mathrm{ml}^{-1}$ in sterile 96 -well microtiter plates containing Mueller-Hinton broth (Torlak, Belgrade, Serbia). The final volume in each well was $100 \mu \mathrm{l}$. After that, $10 \mu \mathrm{l}$ of each diluted bacterial suspension was added to appropriate wells. The inoculated plates were incubated at $37^{\circ} \mathrm{C}$ for $24 \mathrm{~h}$. The results were evaluated by observing broth turbidity. MIC was defined as the lowest concentration of tested substance that inhibited bacterial growth (no broth turbidity). Each test included growth control and sterility control. All tests were performed in duplicate and MICs were constant.

\subsection{Effects of preservatives on sugar fermentation}

The effects of preservatives on fermentative ability of bacteria were tested using carbohydrate test. Five millilitres of Andrade peptone water with Durham tube containing 1\% sugar (glucose, dextrose, or lactose (Torlak, Belgrade, Serbia)) and the tested preservative at MIC concentration and sub-inhibitory concentration (1/2 MIC), was inoculated with $10 \mu \mathrm{l}$ of bacterial suspension corresponding to McFarland standard No. 0.5. After the incubation at 37 ${ }^{\circ} \mathrm{C}$ for $24 \mathrm{~h}$, the results were evaluated by observing colour change of Andrade indicator (acid production) and the production or absence of gas in Durham tubes. After treatment with MIC concentrations of preservatives, the growth of bacteria was confirmed by inoculation on agar 
slants. This control confirms that the colour of indicator changed due to bacterial activity rather than due to reaction with the preservative. The experiment included also bacterial growth control, sterility control of the nutrient broth, and sterility control of nutrient broth containing preservatives.

\subsection{Effects of preservative on cell membrane permeability}

The release of proteins into the supernatant, as a result of cell membrane permeability, after treatment of bacterial cells with potassium benzoate was investigated. Two probiotic strains, B. subtilis IP 5832 and L. plantarum, as well as four isolates of E. coli, S. enterica, P. mirabilis, and $K$. pneumoniae were used. Overnight bacterial culture was collected by centrifugation at 5000 r.p.m. for $15 \mathrm{~min}$ (Hettich centrifuge, Micro120, Germany) and washed three times using phosphate buffered saline (PBS, pH 7.4) (Alfa Aesar GmbH \& Co. Karlsruhe, Germany). Bacterial cells were re-suspended in PBS containing potassium benzoate in subinhibitory concentration (1/2 MIC) and incubated at $37^{\circ} \mathrm{C}$ for $16-18 \mathrm{~h}$. After incubation, the samples were centrifuged at 5000 r.p.m. for $10 \mathrm{~min}$. The concentration of protein in the supernatant was determined using Bradford reagent (AppliChem GmbH., Germany) (BRADFORD, 1976). The absorbances were monitored with a spectrophotometer at $\lambda=595$. Untreated samples (bacteria + PBS) and tested compounds (preservatives + PBS) served as controls. The antibiotic - polymixin B (Sigma-Aldrich INC., USA) $\left(20 \mu \mathrm{g} \mathrm{ml}^{-1}\right)$ was used as positive control. The experiment was performed in triplicate and mean values were presented.

\subsection{Effects of preservative on amylolytic activity}

The potential influence of potassium benzoate on amylolytic activity of starch degrading bacteria (B. subtilis IP 5832, B. subtilis ATCC 6633, B. subtilis, and B. cereus) was investigated by spectrophotometric method according to HASAN and co-workers (2013) with modifications. Sterile mineral broth $(10 \mathrm{ml})$ with $1 \%$ starch containing the potassium benzoate at subinhibitory concentration (1/2 MIC) was inoculated with $100 \mu \mathrm{l}$ of bacterial suspension (McFarland standard No. 0.5). After incubation at $37^{\circ} \mathrm{C}$ for $24 \mathrm{~h}$, the samples were centrifuged at 5000 r.p.m. for 20 min (Hettich centrifuge, Micro120, Germany). The supernatants were used as enzyme extract. One millilitre of the obtained extract was mixed with $1 \mathrm{ml}$ of dissolved starch and incubated at $37^{\circ} \mathrm{C}$ for $15 \mathrm{~min}$. After incubation, $0.5 \mathrm{ml}$ of Lugol solution and $6 \mathrm{ml}$ of distilled water was added. The enzyme activity was measured with a spectrophotometer at $\lambda=540 \mathrm{~nm}$. The experiment was performed in triplicate and mean values were presented. Potassium benzoate (conc. $3.12 \mathrm{mg} \mathrm{ml}^{-1}$ ) was used as control and bacteria that is not capable of starch degradation - Escherichia coli) was used like negative control.

\subsection{Data analysis}

For comparison of treated and untreated samples, used to determine the protein leakage and amylolytic activity of bacteria, data were analysed by the paired samples $t$-test, using SPSS 20 package (SPSS Inc., Chicago, IL, USA).

\section{Results and discussion}

In this paper, tested preservatives demonstrated different in vitro antimicrobial activity on food borne bacteria (Table 1). Results of antimicrobial activity of potassium propionate are 
presented here for the first time. Potassium metabisulphite demonstrated the highest inhibitory activity. MIC varied in the range of $0.78 \mathrm{mg} \mathrm{ml}^{-1}$ to $3.12 \mathrm{mg} \mathrm{ml}^{-1}$. Potassium nitrate showed the lowest inhibitory activity.

Table 1. Antibacterial activity of tested preservatives $\left(\mathrm{mg} \mathrm{ml}^{-1}\right)$

\begin{tabular}{|c|c|c|c|c|}
\hline \multirow[t]{2}{*}{ Species } & $\begin{array}{c}\text { Potassium } \\
\text { metabisulphite }\end{array}$ & $\begin{array}{c}\text { Potassium } \\
\text { benzoate }\end{array}$ & $\begin{array}{l}\text { Potassium } \\
\text { propionate }\end{array}$ & $\begin{array}{c}\text { Potassium } \\
\text { nitrate }\end{array}$ \\
\hline & \multicolumn{4}{|c|}{ MIC } \\
\hline E. coli ATCC 25922 & 3.12 & 12.5 & 12.5 & 12.5 \\
\hline E. coli & 3.12 & 12.5 & 12.5 & 12.5 \\
\hline S. enterica & 3.12 & 12.5 & 12.5 & 12.5 \\
\hline S. typhimurium & 1.56 & 6.25 & 50 & 100 \\
\hline K. pneumoniae ATCC 70063 & 1.56 & 6.25 & 25 & 100 \\
\hline K. pneumoniae & 1.56 & 6.25 & 25 & 100 \\
\hline P. mirabilis & 1.56 & 6.25 & 50 & 100 \\
\hline Staphylococcus sp. & 1.56 & 6.25 & 50 & 100 \\
\hline P. aeruginosa & 0.78 & 6.25 & 6.25 & 50 \\
\hline B. cereus & 0.78 & 6.25 & 50 & 100 \\
\hline B. subtilis & 1.56 & 3.12 & 25 & 100 \\
\hline B. subtilis ATCC 6633 & 1.56 & 6.25 & 50 & 100 \\
\hline B. subtilis IP 5832 & 1.56 & 12.5 & 6.25 & 6.25 \\
\hline B. animalis subsp. lactis & 0.78 & 6.25 & 50 & 100 \\
\hline L. plantarum & 1.56 & 6.25 & 100 & 100 \\
\hline
\end{tabular}

MIC: minimum inhibitory concentrations

OLADAPO and co-workers (2014) reported that potassium metabisulphite had the highest antimicrobial activity among tested preservatives. The appropriate combination of nisin (subinhibitory concentration) and potassium metabisulphite (MIC concentration) inhibit the growth of lactic acid bacteria (Rojo-BEZARES et al., 2007). The effect of sodium benzoate, sodium nitrite, and potassium sorbate at producing antimicrobial packaging material was investigated by VARTIAINEN and co-workers (2003). Authors have noticed that plaques containing $15 \%$ of sodium nitrite, sodium benzoate, and potassium sorbate inhibited growth of $B$. subtilis. This study confirmed the inhibitory activity of benzoate and nitrate salts against some bacteria causing food spoilage.

\subsection{Effects of preservatives on sugar fermentation}

The effects of preservatives on bacterial fermentation of glucose, dextrose, and lactose were evaluated by acid and gas production (Tables 2, 3, and 4). The effect of potassium metabisulphite is not shown, because the compound discoloured the Andrade indicator.

Potassium benzoate demonstrated the strongest inhibitory effect, while potassium propionate and potassium nitrate demonstrated fair inhibitory effect on sugar fermentation. The tested sugars were fermented by E. coli ATCC 25922, E. coli, K. pneumoniae ATCC 70063, and K. pneumoniae. S. enterica, S. typhimurium, P. mirabilis, and Staphylococcus sp. demonstrated the ability of glucose and dextrose fermentation by gas and acid production. Lactose was not fermented by the tested bacteria. B. cereus, B. subtilis, B. subtilis ATCC 
6633, B. subtilis IP 5832, B. animalis subsp. lactis, and L. plantarum fermented glucose and dextrose with production of acid but no gas. Lactose could only be fermented by $B$. subtilis IP5832. At MIC, fermentation of both sugars was interrupted, while incomplete/partial fermentation without gas production was observed at the sub-inhibitory concentrations.

Table 2. Effect of potassium benzoate on sugar fermentation

\begin{tabular}{|c|c|c|c|c|c|c|c|c|c|}
\hline & \multicolumn{9}{|c|}{ Potassium benzoate } \\
\hline & \multicolumn{3}{|c|}{$\mathrm{MIC}$} & \multicolumn{3}{|c|}{$1 / 2 \mathrm{MIC}$} & \multicolumn{3}{|c|}{ Growth control } \\
\hline & Glucose & Lactose & Dextrose & Glucose & Lactose & Dextrose & Glucose & Lactose & Dextrose \\
\hline E. coli ATCC & A- & A- & A- & $\mathrm{A}-$ & A- & $\mathrm{A}-$ & $\mathrm{A}+$ & $\mathrm{A}+$ & $\mathrm{A}+$ \\
\hline 25922 & G- & G- & G- & G- & G- & $\mathrm{G}-$ & $\mathrm{G}^{+}$ & $\mathrm{G}+$ & $\mathrm{G}^{+}$ \\
\hline E. coli & $\begin{array}{l}\mathrm{A}- \\
\mathrm{G}-\end{array}$ & $\begin{array}{l}\mathrm{A}- \\
\mathrm{G}-\end{array}$ & $\begin{array}{l}\mathrm{A}- \\
\mathrm{G}-\end{array}$ & $\begin{array}{l}\mathrm{A}- \\
\mathrm{G}-\end{array}$ & $\begin{array}{l}\mathrm{A}- \\
\mathrm{G}-\end{array}$ & $\begin{array}{l}\mathrm{A}- \\
\mathrm{G}-\end{array}$ & $\begin{array}{l}\mathrm{A}+ \\
\mathrm{G}+\end{array}$ & $\begin{array}{l}\mathrm{A}+ \\
\mathrm{G}+\end{array}$ & $\begin{array}{l}\mathrm{A}+ \\
\mathrm{G}+\end{array}$ \\
\hline S. enterica & $\begin{array}{l}\mathrm{A}- \\
\mathrm{G}-\end{array}$ & n.f. & $\begin{array}{l}\mathrm{A}- \\
\mathrm{G}-\end{array}$ & $\begin{array}{c}\mathrm{Aw}^{+} \\
\mathrm{G}-\end{array}$ & n.f. & $\begin{array}{c}\mathrm{Aw}^{+} \\
\mathrm{G}-\end{array}$ & $\begin{array}{l}\mathrm{A}+ \\
\mathrm{G}+\end{array}$ & n.f. & $\begin{array}{l}\mathrm{A}+ \\
\mathrm{G}+\end{array}$ \\
\hline S. typhimurium & $\begin{array}{l}\mathrm{A}- \\
\mathrm{G}-\end{array}$ & n.f. & $\begin{array}{l}\mathrm{A}- \\
\mathrm{G}-\end{array}$ & $\begin{array}{l}\mathrm{Aw}+ \\
\mathrm{G}-\end{array}$ & n.f. & $\begin{array}{l}\mathrm{Aw}^{+} \\
\mathrm{G}-\end{array}$ & $\begin{array}{l}\mathrm{A}+ \\
\mathrm{G}+\end{array}$ & n.f. & $\begin{array}{l}\mathrm{A}+ \\
\mathrm{G}+\end{array}$ \\
\hline $\begin{array}{l}\text { K. pneumoniae } \\
\text { ATCC } 70063\end{array}$ & $\begin{array}{l}\mathrm{A}- \\
\mathrm{G}-\end{array}$ & $\begin{array}{l}\mathrm{A}- \\
\mathrm{G}-\end{array}$ & $\begin{array}{l}\mathrm{A}- \\
\mathrm{G}-\end{array}$ & $\begin{array}{l}\mathrm{A}- \\
\mathrm{G}-\end{array}$ & $\begin{array}{l}\mathrm{A}- \\
\mathrm{G}-\end{array}$ & $\begin{array}{l}\mathrm{A}- \\
\mathrm{G}-\end{array}$ & $\begin{array}{l}\mathrm{A}+ \\
\mathrm{G}+\end{array}$ & $\begin{array}{l}\mathrm{A}+ \\
\mathrm{G}+\end{array}$ & $\begin{array}{l}\mathrm{A}+ \\
\mathrm{G}+\end{array}$ \\
\hline$K$. pneumoniae & $\begin{array}{l}\mathrm{A}- \\
\mathrm{G}-\end{array}$ & $\begin{array}{l}\mathrm{A}- \\
\mathrm{G}-\end{array}$ & $\begin{array}{l}\mathrm{A}- \\
\mathrm{G}-\end{array}$ & $\begin{array}{l}\mathrm{A}- \\
\mathrm{G}-\end{array}$ & $\begin{array}{l}\mathrm{A}- \\
\mathrm{G}-\end{array}$ & $\begin{array}{l}\mathrm{A}- \\
\mathrm{G}-\end{array}$ & $\begin{array}{l}\mathrm{A}+ \\
\mathrm{G}+\end{array}$ & $\begin{array}{l}\mathrm{A}+ \\
\mathrm{G}+\end{array}$ & $\begin{array}{l}\mathrm{A}+ \\
\mathrm{G}+\end{array}$ \\
\hline P. mirabilis & $\begin{array}{l}\mathrm{A}- \\
\mathrm{G}-\end{array}$ & n.f. & $\begin{array}{l}\mathrm{A}- \\
\mathrm{G}-\end{array}$ & $\begin{array}{c}\mathrm{Aw}+ \\
\mathrm{G}-\end{array}$ & n.f. & $\begin{array}{c}\mathrm{Aw}^{+} \\
\mathrm{G}-\end{array}$ & $\begin{array}{l}\mathrm{A}+ \\
\mathrm{G}+\end{array}$ & n.f. & $\begin{array}{l}\mathrm{A}+ \\
\mathrm{G}^{+}\end{array}$ \\
\hline $\begin{array}{l}\text { Staphylococcus } \\
\text { sp. }\end{array}$ & $\begin{array}{l}\mathrm{A}- \\
\mathrm{G}-\end{array}$ & $\begin{array}{l}\mathrm{A}- \\
\mathrm{G}-\end{array}$ & $\begin{array}{l}\mathrm{A}- \\
\mathrm{G}-\end{array}$ & $\begin{array}{c}\mathrm{Aw}+ \\
\mathrm{G}-\end{array}$ & $\begin{array}{c}\mathrm{Aw}^{+} \\
\mathrm{G}-\end{array}$ & $\begin{array}{c}\mathrm{Aw}+ \\
\mathrm{G}-\end{array}$ & $\begin{array}{l}\mathrm{A}+ \\
\mathrm{G}+\end{array}$ & $\begin{array}{c}\mathrm{Aw}+ \\
\mathrm{G}-\end{array}$ & $\begin{array}{l}\mathrm{A}+ \\
\mathrm{G}+\end{array}$ \\
\hline P. aeruginosa & $\begin{array}{l}\mathrm{A}- \\
\mathrm{G}-\end{array}$ & n.f. & $\begin{array}{l}\mathrm{A}- \\
\mathrm{G}-\end{array}$ & $\begin{array}{c}\mathrm{Aw}+ \\
\mathrm{G}-\end{array}$ & n.f. & $\begin{array}{c}\mathrm{Aw}^{+} \\
\mathrm{G}-\end{array}$ & $\begin{array}{l}\mathrm{A}+ \\
\mathrm{G}-\end{array}$ & n.f. & $\begin{array}{l}\mathrm{A}+ \\
\mathrm{G}-\end{array}$ \\
\hline B. cereus & $\begin{array}{l}\mathrm{A}- \\
\mathrm{G}-\end{array}$ & n.f. & $\begin{array}{l}\mathrm{A}- \\
\mathrm{G}-\end{array}$ & $\begin{array}{l}\mathrm{A}- \\
\mathrm{G}-\end{array}$ & n.f. & $\begin{array}{l}\mathrm{A}- \\
\mathrm{G}-\end{array}$ & $\begin{array}{l}\mathrm{A}+ \\
\mathrm{G}-\end{array}$ & n.f. & $\begin{array}{l}\mathrm{A}+ \\
\mathrm{G}-\end{array}$ \\
\hline B. subtilis & $\begin{array}{l}\mathrm{A}- \\
\mathrm{G}-\end{array}$ & n.f. & $\begin{array}{l}\mathrm{A}- \\
\mathrm{G}-\end{array}$ & $\begin{array}{l}\mathrm{A}- \\
\mathrm{G}-\end{array}$ & n.f. & $\begin{array}{l}\mathrm{A}- \\
\mathrm{G}-\end{array}$ & $\begin{array}{l}\mathrm{A}+ \\
\mathrm{G}-\end{array}$ & n.f. & $\begin{array}{l}\mathrm{A}+ \\
\mathrm{G}-\end{array}$ \\
\hline $\begin{array}{l}\text { B. subtilis } \\
\text { ATCC } 6633\end{array}$ & $\begin{array}{l}\mathrm{A}- \\
\mathrm{G}-\end{array}$ & n.f. & $\begin{array}{l}\mathrm{A}- \\
\mathrm{G}-\end{array}$ & $\begin{array}{c}\mathrm{Aw}^{+} \\
\mathrm{G}-\end{array}$ & n.f. & $\begin{array}{c}\mathrm{Aw}^{+} \\
\mathrm{G}-\end{array}$ & $\begin{array}{l}\mathrm{A}+ \\
\mathrm{G}-\end{array}$ & n.f. & $\begin{array}{l}\mathrm{A}+ \\
\mathrm{G}-\end{array}$ \\
\hline $\begin{array}{l}\text { B. subtilis IP } \\
5832\end{array}$ & $\begin{array}{l}\mathrm{A}- \\
\mathrm{G}-\end{array}$ & n.f. & $\begin{array}{l}\mathrm{A}- \\
\mathrm{G}-\end{array}$ & $\begin{array}{c}\mathrm{Aw}^{+} \\
\mathrm{G}-\end{array}$ & n.f. & $\begin{array}{c}\mathrm{Aw}^{+} \\
\mathrm{G}-\end{array}$ & $\begin{array}{l}\mathrm{A}+ \\
\mathrm{G}-\end{array}$ & $\begin{array}{l}\mathrm{A}+ \\
\mathrm{G}-\end{array}$ & $\begin{array}{l}\mathrm{A}+ \\
\mathrm{G}-\end{array}$ \\
\hline $\begin{array}{l}\text { B. animalis } \\
\text { subsp.lactis }\end{array}$ & $\begin{array}{l}\mathrm{A}- \\
\mathrm{G}-\end{array}$ & n.f. & $\begin{array}{l}\mathrm{A}- \\
\mathrm{G}-\end{array}$ & $\begin{array}{c}\mathrm{Aw}+ \\
\mathrm{G}-\end{array}$ & n.f. & $\begin{array}{c}\mathrm{Aw}^{+} \\
\mathrm{G}-\end{array}$ & $\begin{array}{l}\mathrm{A}+ \\
\mathrm{G}-\end{array}$ & n.f. & $\begin{array}{l}\mathrm{A}+ \\
\mathrm{G}-\end{array}$ \\
\hline L. plantarum & $\begin{array}{l}\mathrm{A}- \\
\mathrm{G}-\end{array}$ & n.f. & $\begin{array}{l}\mathrm{A}- \\
\mathrm{G}-\end{array}$ & $\begin{array}{l}\mathrm{A}- \\
\mathrm{G}-\end{array}$ & n.f. & $\begin{array}{l}\mathrm{A}- \\
\mathrm{G}-\end{array}$ & $\begin{array}{l}\mathrm{A}+ \\
\mathrm{G}-\end{array}$ & n.f. & $\begin{array}{l}\mathrm{A}+ \\
\mathrm{G}-\end{array}$ \\
\hline
\end{tabular}

+: Positive; -: negative; w+: weakly positive; A: acid production; G: gas production; n.f.: no fermentation 
Table 3. Effect of potassium nitrate on sugar fermentation

\begin{tabular}{|c|c|c|c|c|c|c|c|c|c|}
\hline & \multicolumn{9}{|c|}{ Potassium nitrate } \\
\hline & \multicolumn{3}{|c|}{$\mathrm{MIC}$} & \multicolumn{3}{|c|}{$1 / 2 \mathrm{MIC}$} & \multicolumn{3}{|c|}{ Growth control } \\
\hline & Glucose & Lactose & Dextrose & Glucose & Lactose & Dextrose & Glucose & Lactose & Dextrose \\
\hline$\overline{E \text { E. coli ATCC }}$ & $\mathrm{A}_{-}$ & A- & $\mathrm{A}_{-}$ & A- & $\mathrm{A}_{-}$ & A- & $\mathrm{A}+$ & $\mathrm{A}+$ & $\mathrm{A}+$ \\
\hline 25922 & G- & G- & G- & G- & $\mathrm{G}_{-}$ & G- & $\mathrm{G}^{+}$ & $\mathrm{G}^{+}$ & $\mathrm{G}^{+}$ \\
\hline \multirow{2}{*}{ E. coli } & $\mathrm{A}_{-}$ & $\mathrm{A}_{-}$ & A- & $\mathrm{A} \pm$ & $\mathrm{A}-$ & $\mathrm{A}-$ & $\mathrm{A}+$ & $\mathrm{A}+$ & $\mathrm{A}+$ \\
\hline & G- & $\mathrm{G}_{-}$ & $\mathrm{G}-$ & G- & G- & $\mathrm{G}_{-}$ & $\mathrm{G}^{+}$ & $\mathrm{G}^{+}$ & $\mathrm{G}^{+}$ \\
\hline \multirow{2}{*}{ S. enterica } & $\mathrm{A}_{-}$ & n.f. & $\mathrm{A}_{-}$ & $\mathrm{A}_{-}$ & n.f. & $\mathrm{A}-$ & $\mathrm{A}+$ & n.f. & $\mathrm{A}+$ \\
\hline & G- & & G- & G- & & $\mathrm{G}_{-}$ & $\mathrm{G}^{+}$ & & $\mathrm{G}^{+}$ \\
\hline \multirow{2}{*}{ S. typhimurium } & $\mathrm{A}_{-}$ & n.f. & $\mathrm{A}-$ & $\mathrm{Aw}^{+}$ & n.f. & $\mathrm{Aw}^{+}$ & $\mathrm{A}+$ & n.f. & $\mathrm{A}+$ \\
\hline & G- & & G- & $\mathrm{G}-$ & & $\mathrm{G}-$ & $\mathrm{G}^{+}$ & & $\mathrm{G}^{+}$ \\
\hline K. pneumoniae & $\mathrm{A}_{-}$ & $\mathrm{A}-$ & A- & $\mathrm{Aw}+$ & $\mathrm{Aw}+$ & $\mathrm{Aw}+$ & $\mathrm{A}+$ & $\mathrm{A}+$ & $\mathrm{A}+$ \\
\hline ATCC 70063 & G- & G- & G- & $\mathrm{G}-$ & $\mathrm{G}-$ & $\mathrm{G}-$ & $\mathrm{G}^{+}$ & $\mathrm{G}^{+}$ & $\mathrm{G}^{+}$ \\
\hline \multirow{2}{*}{ K. pneumoniae } & $\mathrm{A}_{-}$ & $\mathrm{A}_{-}$ & $\mathrm{A}-$ & $\mathrm{Aw}+$ & $\mathrm{Aw}+$ & $\mathrm{Aw}+$ & $\mathrm{A}+$ & $\mathrm{A}+$ & $\mathrm{A}+$ \\
\hline & G- & G- & G- & $\mathrm{G}-$ & $\mathrm{G}-$ & $\mathrm{G}-$ & $\mathrm{G}^{+}$ & $\mathrm{G}^{+}$ & $\mathrm{G}^{+}$ \\
\hline \multirow{2}{*}{ P. mirabilis } & $\mathrm{A}_{-}$ & n.f. & $\mathrm{A}-$ & $\mathrm{Aw}^{+}$ & n.f. & $\mathrm{Aw}^{+}$ & $\mathrm{A}+$ & n.f. & $\mathrm{A}+$ \\
\hline & G- & & G- & $\mathrm{G}-$ & & $\mathrm{G}-$ & $\mathrm{G}^{+}$ & & $\mathrm{G}^{+}$ \\
\hline \multirow{2}{*}{$\begin{array}{l}\text { Staphylococcus } \\
\text { sp. }\end{array}$} & $\mathrm{Aw}^{+}$ & $\mathrm{A}_{-}$ & $\mathrm{A}_{-}$ & $\mathrm{Aw}+$ & Aw + & Aw + & $\mathrm{A}+$ & $\mathrm{Aw}+$ & $\mathrm{A}+$ \\
\hline & $\mathrm{G}-$ & $\mathrm{G}_{-}$ & G- & $\mathrm{G}-$ & G- & $\mathrm{G}-$ & $\mathrm{G}^{+}$ & $\mathrm{G}-$ & $\mathrm{G}^{+}$ \\
\hline \multirow{2}{*}{ P. aeruginosa } & $\mathrm{A}-$ & n.f. & A- & Aw + & n.f. & $\mathrm{Aw}+$ & $\mathrm{A}+$ & n.f. & $\mathrm{A}+$ \\
\hline & G- & & G- & $\mathrm{G}-$ & & $\mathrm{G}-$ & G- & & G- \\
\hline \multirow{2}{*}{ B. cereus } & $\mathrm{A}_{-}$ & n.f. & $\mathrm{A}_{-}$ & $\mathrm{A}_{-}$ & n.f. & $\mathrm{A}-$ & $\mathrm{A}+$ & n.f. & $\mathrm{A}+$ \\
\hline & G- & & G- & G- & & G- & G- & & G- \\
\hline \multirow{2}{*}{ B. subtilis } & $\mathrm{A}_{-}$ & n.f. & $\mathrm{A}-$ & $\mathrm{A}_{-}$ & n.f. & $\mathrm{A}_{-}$ & $\mathrm{A}+$ & n.f. & $\mathrm{A}+$ \\
\hline & G- & & $\mathrm{G}-$ & G- & & $\mathrm{G}-$ & $\mathrm{G}-$ & & $\mathrm{G}-$ \\
\hline B. subtilis & $\mathrm{A}_{-}$ & n.f. & $\mathrm{A}-$ & $\mathrm{A}_{-}$ & n.f. & $\mathrm{A}-$ & $\mathrm{A}+$ & n.f. & $\mathrm{A}+$ \\
\hline ATCC 6633 & G- & & $\mathrm{G}-$ & $\mathrm{G}-$ & & $\mathrm{G}-$ & $\mathrm{G}-$ & & $\mathrm{G}-$ \\
\hline \multirow{2}{*}{$\begin{array}{l}\text { B. subtilis IP } \\
5832\end{array}$} & $\mathrm{~A}_{-}$ & n.f. & $\mathrm{A}-$ & $\mathrm{Aw}+$ & n.f. & Aw+ & $\mathrm{A}+$ & $\mathrm{A}+$ & $\mathrm{A}+$ \\
\hline & G- & & G- & $\mathrm{G}-$ & & $\mathrm{G}-$ & G- & G- & G- \\
\hline \multirow{2}{*}{$\begin{array}{l}\text { B. animalis } \\
\text { subsp. lactis }\end{array}$} & $\mathrm{A}-$ & n.f. & $\mathrm{A}-$ & Aw + & n.f. & $\mathrm{Aw}^{+}$ & $\mathrm{A}+$ & n.f. & $\mathrm{A}+$ \\
\hline & G- & & $\mathrm{G}_{-}$ & $\mathrm{G}-$ & & G- & G- & & G- \\
\hline \multirow{2}{*}{ L. plantarum } & $\mathrm{Aw}^{+}$ & n.f. & $\mathrm{Aw}+$ & $\mathrm{Aw}+$ & n.f. & Aw + & $\mathrm{A}+$ & n.f. & $\mathrm{A}+$ \\
\hline & $\mathrm{G}-$ & & $\mathrm{G}-$ & $\mathrm{G}-$ & & $\mathrm{G}-$ & G- & & G- \\
\hline
\end{tabular}

+: Positive; -: negative; w+: weakly positive; A: acid production; G: gas production; n.f.: no fermentation 
Table 4. Effect of potassium propionate on sugar fermentation

\begin{tabular}{|c|c|c|c|c|c|c|c|c|c|}
\hline & \multicolumn{9}{|c|}{ Potassium propionate } \\
\hline & \multicolumn{3}{|c|}{ MIC } & \multicolumn{3}{|c|}{$1 / 2 \mathrm{MIC}$} & \multicolumn{3}{|c|}{ Growth control } \\
\hline & Glucose & Lactose & Dextrose & Glucose & Lactose & Dextrose & Glucose & Lactose & Dextrose \\
\hline E. coli ATCC & $\mathrm{A}-$ & A- & $\mathrm{A}_{-}$ & Aw + & Aw + & $\mathrm{Aw}+$ & $\mathrm{A}+$ & $\mathrm{A}+$ & $\mathrm{A}+$ \\
\hline 25922 & G- & $\mathrm{G}-$ & $\mathrm{G}-$ & G- & G- & $\mathrm{G}-$ & $\mathrm{G}+$ & $\mathrm{G}+$ & $\mathrm{G}+$ \\
\hline E. coli & $\begin{array}{l}\mathrm{A}- \\
\mathrm{G}-\end{array}$ & $\begin{array}{l}\mathrm{A}- \\
\mathrm{G}-\end{array}$ & $\begin{array}{l}\mathrm{A}- \\
\mathrm{G}-\end{array}$ & $\begin{array}{l}\mathrm{Aw}^{+} \\
\mathrm{G}-\end{array}$ & $\begin{array}{c}\mathrm{Aw}^{+} \\
\mathrm{G}-\end{array}$ & $\begin{array}{c}\mathrm{Aw}^{+} \\
\mathrm{G}-\end{array}$ & $\begin{array}{l}\mathrm{A}+ \\
\mathrm{G}+\end{array}$ & $\begin{array}{l}\mathrm{A}+ \\
\mathrm{G}^{+}\end{array}$ & $\begin{array}{l}\mathrm{A}+ \\
\mathrm{G}+\end{array}$ \\
\hline S. enterica & $\begin{array}{c}\mathrm{Aw}+ \\
\mathrm{G}-\end{array}$ & n.f. & $\begin{array}{c}\mathrm{A}+\mathrm{w} \\
\mathrm{G}-\end{array}$ & $\begin{array}{l}\mathrm{Aw}^{+} \\
\mathrm{G}-\end{array}$ & n.f. & $\begin{array}{c}\mathrm{Aw}^{+} \\
\mathrm{G}-\end{array}$ & $\begin{array}{l}\mathrm{A}+ \\
\mathrm{G}+\end{array}$ & n.f. & $\begin{array}{l}\mathrm{A}+ \\
\mathrm{G}+\end{array}$ \\
\hline S. typhimurium & $\begin{array}{c}\mathrm{Aw}+ \\
\mathrm{G}-\end{array}$ & n.f. & $\begin{array}{c}\mathrm{A}+\mathrm{W} \\
\mathrm{G}-\end{array}$ & $\begin{array}{c}\mathrm{Aw}^{+} \\
\mathrm{G}-\end{array}$ & n.f. & $\begin{array}{c}\mathrm{Aw}^{+} \\
\mathrm{G}-\end{array}$ & $\begin{array}{l}\mathrm{A}+ \\
\mathrm{G}+\end{array}$ & n.f. & $\begin{array}{l}\mathrm{A}+ \\
\mathrm{G}^{+}\end{array}$ \\
\hline $\begin{array}{l}\text { K. pneumoniae } \\
\text { ATCC } 70063\end{array}$ & $\begin{array}{l}\mathrm{A}- \\
\mathrm{G}-\end{array}$ & $\begin{array}{l}\mathrm{A}- \\
\mathrm{G}-\end{array}$ & $\begin{array}{l}\mathrm{A}- \\
\mathrm{G}-\end{array}$ & $\begin{array}{c}\mathrm{Aw}+ \\
\mathrm{G}-\end{array}$ & $\begin{array}{c}\mathrm{Aw}+ \\
\mathrm{G}-\end{array}$ & $\begin{array}{c}\mathrm{Aw}+ \\
\mathrm{G}-\end{array}$ & $\begin{array}{l}\mathrm{A}+ \\
\mathrm{G}+\end{array}$ & $\begin{array}{l}\mathrm{A}+ \\
\mathrm{G}+\end{array}$ & $\begin{array}{l}\mathrm{A}+ \\
\mathrm{G}+\end{array}$ \\
\hline K. pneumoniae & $\begin{array}{l}\mathrm{A}- \\
\mathrm{G}-\end{array}$ & $\begin{array}{l}\mathrm{A}- \\
\mathrm{G}-\end{array}$ & $\begin{array}{l}\mathrm{A}- \\
\mathrm{G}-\end{array}$ & $\begin{array}{c}\mathrm{Aw}+ \\
\mathrm{G}-\end{array}$ & $\begin{array}{c}\mathrm{Aw}+ \\
\mathrm{G}-\end{array}$ & $\begin{array}{c}\mathrm{Aw}+ \\
\mathrm{G}-\end{array}$ & $\begin{array}{l}\mathrm{A}+ \\
\mathrm{G}+\end{array}$ & $\begin{array}{l}\mathrm{A}+ \\
\mathrm{G}+\end{array}$ & $\begin{array}{l}\mathrm{A}+ \\
\mathrm{G}+\end{array}$ \\
\hline P. mirabilis & $\begin{array}{l}\mathrm{A}- \\
\mathrm{G}-\end{array}$ & n.f. & $\begin{array}{l}\mathrm{A}- \\
\mathrm{G}-\end{array}$ & $\begin{array}{l}\mathrm{Aw}+ \\
\mathrm{G}-\end{array}$ & n.f. & $\begin{array}{l}\mathrm{Aw}^{+} \\
\mathrm{G}-\end{array}$ & $\begin{array}{l}\mathrm{A}+ \\
\mathrm{G}+\end{array}$ & n.f. & $\begin{array}{l}\mathrm{A}+ \\
\mathrm{G}+\end{array}$ \\
\hline $\begin{array}{l}\text { Staphylococcus } \\
\text { sp. }\end{array}$ & $\begin{array}{l}\mathrm{A}- \\
\mathrm{G}-\end{array}$ & $\begin{array}{l}\mathrm{A}- \\
\mathrm{G}-\end{array}$ & $\begin{array}{l}\mathrm{A}- \\
\mathrm{G}-\end{array}$ & $\begin{array}{c}\mathrm{Aw}^{+} \\
\mathrm{G}-\end{array}$ & $\begin{array}{c}\mathrm{A}+\mathrm{W} \\
\mathrm{G}-\end{array}$ & $\begin{array}{c}\mathrm{Aw}^{+} \\
\mathrm{G}-\end{array}$ & $\begin{array}{l}\mathrm{A}+ \\
\mathrm{G}+\end{array}$ & $\begin{array}{c}\mathrm{Aw}^{+} \\
\mathrm{G}-\end{array}$ & $\begin{array}{l}\mathrm{A}+ \\
\mathrm{G}+\end{array}$ \\
\hline P. aeruginosa & $\begin{array}{l}\mathrm{A}- \\
\mathrm{G}-\end{array}$ & n.f. & $\begin{array}{l}\mathrm{A}- \\
\mathrm{G}-\end{array}$ & $\begin{array}{c}\mathrm{Aw}+ \\
\mathrm{G}-\end{array}$ & n.f. & $\begin{array}{c}\mathrm{Aw}+ \\
\mathrm{G}-\end{array}$ & $\begin{array}{l}\mathrm{A}+ \\
\mathrm{G}-\end{array}$ & n.f. & $\begin{array}{l}\mathrm{A}+ \\
\mathrm{G}-\end{array}$ \\
\hline B. cereus & $\begin{array}{l}\mathrm{A}- \\
\mathrm{G}-\end{array}$ & n.f. & $\begin{array}{l}\mathrm{A}- \\
\mathrm{G}-\end{array}$ & $\begin{array}{l}\mathrm{A}- \\
\mathrm{G}-\end{array}$ & n.f. & $\begin{array}{l}\mathrm{A}- \\
\mathrm{G}-\end{array}$ & $\begin{array}{l}\mathrm{A}+ \\
\mathrm{G}-\end{array}$ & n.f. & $\begin{array}{l}\mathrm{A}+ \\
\mathrm{G}-\end{array}$ \\
\hline B. subtilis & $\begin{array}{l}\mathrm{A}- \\
\mathrm{G}-\end{array}$ & n.f. & $\begin{array}{l}\mathrm{A}- \\
\mathrm{G}-\end{array}$ & $\begin{array}{l}\mathrm{A}- \\
\mathrm{G}-\end{array}$ & n.f. & $\begin{array}{l}\mathrm{A}- \\
\mathrm{G}-\end{array}$ & $\begin{array}{l}\mathrm{A}+ \\
\mathrm{G}-\end{array}$ & n.f. & $\begin{array}{l}\mathrm{A}+ \\
\mathrm{G}-\end{array}$ \\
\hline $\begin{array}{l}\text { B. subtilis } \\
\text { ATCC } 6633\end{array}$ & $\begin{array}{l}\mathrm{A}- \\
\mathrm{G}-\end{array}$ & n.f. & $\begin{array}{l}\mathrm{A}- \\
\mathrm{G}-\end{array}$ & $\begin{array}{c}\mathrm{Aw}+ \\
\mathrm{G}-\end{array}$ & n.f. & $\begin{array}{c}\mathrm{Aw}+ \\
\mathrm{G}-\end{array}$ & $\begin{array}{l}\mathrm{A}+ \\
\mathrm{G}-\end{array}$ & n.f. & $\begin{array}{l}\mathrm{A}+ \\
\mathrm{G}-\end{array}$ \\
\hline $\begin{array}{l}\text { B. subtilis IP } \\
5832\end{array}$ & $\begin{array}{l}\mathrm{A}- \\
\mathrm{G}-\end{array}$ & n.f. & $\begin{array}{l}\mathrm{A}- \\
\mathrm{G}-\end{array}$ & $\begin{array}{l}\mathrm{A}- \\
\mathrm{G}-\end{array}$ & n.f. & $\begin{array}{l}\mathrm{A}- \\
\mathrm{G}-\end{array}$ & $\begin{array}{l}\mathrm{A}+ \\
\mathrm{G}-\end{array}$ & $\begin{array}{l}\mathrm{A}+ \\
\mathrm{G}-\end{array}$ & $\begin{array}{l}\mathrm{A}+ \\
\mathrm{G}-\end{array}$ \\
\hline $\begin{array}{l}\text { B. animalis } \\
\text { subsp. lactis }\end{array}$ & $\begin{array}{l}\text { A- } \\
\text { G- }\end{array}$ & n.f. & $\begin{array}{l}\mathrm{A}- \\
\mathrm{G}-\end{array}$ & $\begin{array}{c}\mathrm{Aw}+ \\
\mathrm{G}-\end{array}$ & n.f. & $\begin{array}{c}\mathrm{Aw}+ \\
\mathrm{G}-\end{array}$ & $\begin{array}{l}\mathrm{A}+ \\
\mathrm{G}-\end{array}$ & n.f. & $\begin{array}{l}\mathrm{A}+ \\
\mathrm{G}-\end{array}$ \\
\hline L. plantarum & $\begin{array}{l}\mathrm{A}- \\
\mathrm{G}- \\
\end{array}$ & n.f. & $\begin{array}{l}\mathrm{A}- \\
\mathrm{G}-\end{array}$ & $\begin{array}{c}\mathrm{Aw}^{+} \\
\mathrm{G}-\end{array}$ & n.f. & $\begin{array}{c}\mathrm{Aw}^{+} \\
\mathrm{G}-\end{array}$ & $\begin{array}{l}\mathrm{A}+ \\
\mathrm{G}-\end{array}$ & n.f. & $\begin{array}{l}\mathrm{A}+ \\
\mathrm{G}- \\
\end{array}$ \\
\hline
\end{tabular}

+: Positive; -: negative; w+: weakly positive; A: acid production; G: gas production; n.f.: no fermentation

Potassium benzoate was used as a preservative in non-alcoholic beverages (Sıм, 2001). Also, the influence of potassium propionate and potassium nitrate on glucose fermentation was investigated (BRYANT, 1965; BHASIN \& ModI, 2012). 


\subsection{Effects of preservative on cell membrane permeability}

The results indicated that after adding the sub-inhibitory concentration of potassium benzoate, the protein concentration increased compared to the untreated cells (Fig. 1). The maximum absorbance was observed for E. coli and S. enterica treated cells. For other tested bacteria, the leakage of proteins was not significantly higher than for control samples $(\mathrm{P}>0.05)$. No changes in the absorbance values of treated and untreated (control) cells of $L$. plantarum were observed.

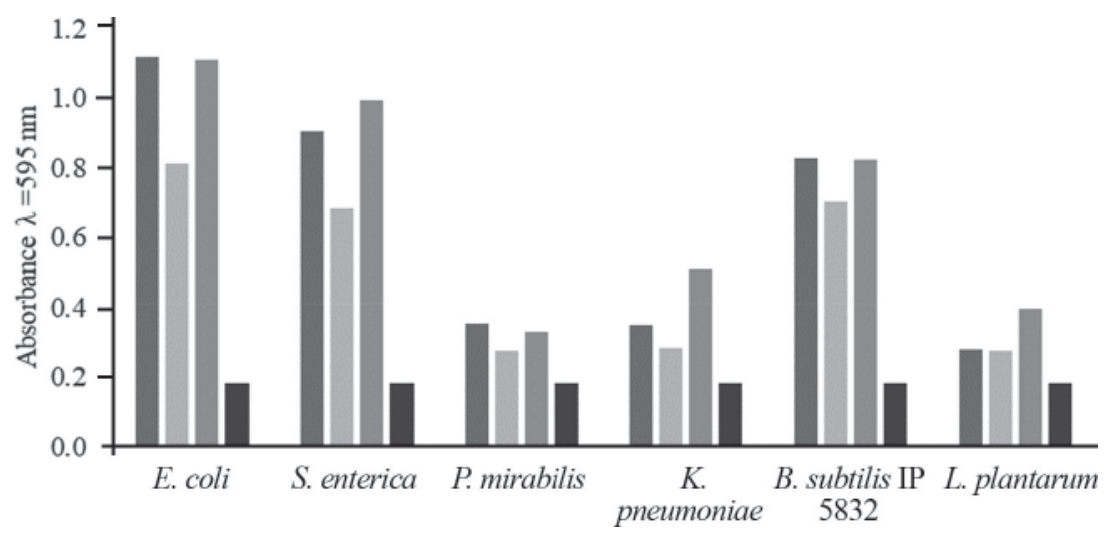

Fig. 1. Effects of preservative on cell membrane permeability (protein leakage)

Treated sample: bacteria + potassium benzoate; untreated sample: bacteria + PBS; positive control: antibiotic polymixin B (conc. $20 \mu \mathrm{g} \mathrm{ml}^{-1}$ ); negative control: potassium benzoate (conc. $3.12 \mathrm{mg} \mathrm{ml}^{-1}$ ).

: Treated sample; : Untreated sample; : Antibiotic; $\square$ : Control

According to the measured protein concentration, potassium benzoate disturbed the integrity and function of cell membranes of bacteria. The cytoplasmic membrane normally acts as a diffusion barrier between the cytoplasm and the extracellular medium (Cox et al., 2001). When cell membrane become compromised, small ions, such as potassium and phosphate, tend to leach out first, followed by large molecules, such as DNA, RNA, proteins, and other materials (DeNYER \& Hugo, 1991). Measurement of specific cell's constituent leakage is an indicative signal of membrane sensitivity to specific antimicrobial agent.

\subsection{Effects of preservative on amylolytic activity}

Bacteria of Bacillus genera are one of the causers of food spoilage (STECCHINI et al., 2013), so in this paper, amylolytic activity inhibition of preservatives was tested. The results indicated that after adding sub-inhibitory concentrations of potassium benzoate, the amylolytic activity was significantly reduced, compared to the untreated cells $(\mathrm{P}<0.05)$ (Fig. 2).

It is widely accepted that undissociated form of benzoate, propionate, and sorbate acts as inhibitors of bacterial growth causing the decrease of intracellular $\mathrm{pH}$. But, these preservatives could be active also in dissociated form, which suggests some other mode of antimicrobial action (EKLund, 1985a, b; PIPER et al., 2001; YAGANZA et al., 2009). The results of this study indicate that possible mechanisms of activity could be inhibition of metabolic enzymes or disturbance of cell membrane function, depending on the type of preservative. 


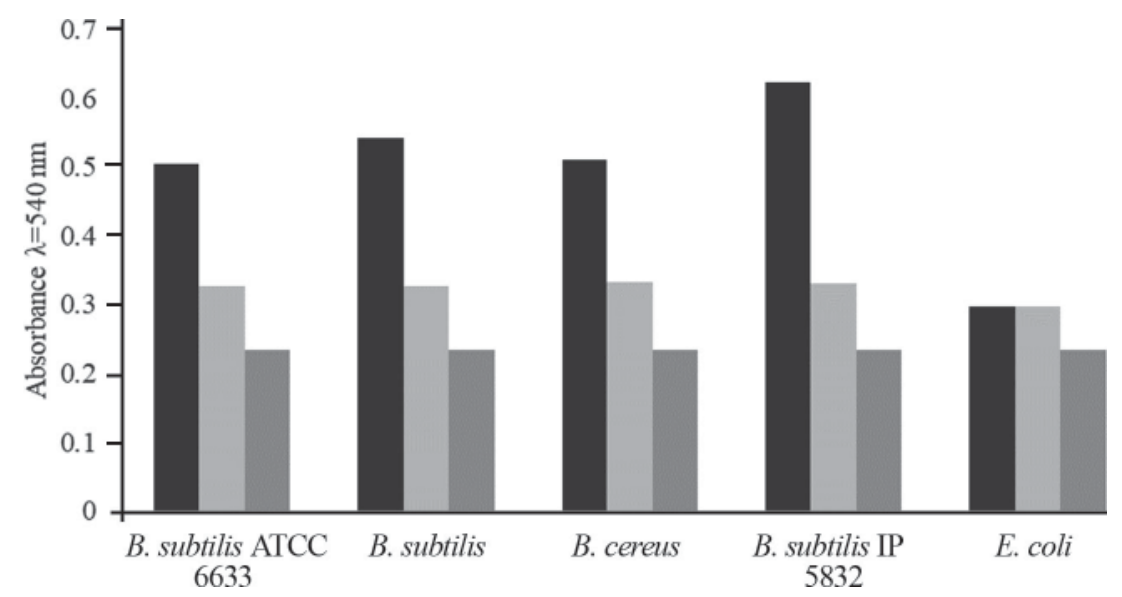

Fig. 2. Effects of preservative on amylolytic activity Treated sample: bacteria + potassium benzoate + mineral broth enriched with $1 \%$ starch; untreated sample: bacteria + mineral broth enriched with $1 \%$ starch; control potassium benzoate (conc. $3.12 \mathrm{mg} \mathrm{m}^{-1}$ ); negative control - E. coli.

$\square$ : Treated sample; : Untreated sample; $\square$ : Control

\section{Conclusions}

Based on the results in our research, it could be concluded that tested preservatives justified their use in control of microorganism's growth. Tested preservatives influenced sugar fermentation of bacteria. In addition, potassium benzoate increased cell membrane permeability and inhibited amylolytic activity of bacteria.

This investigation was supported by the Ministry of Education, Science and Technological Development of the Republic of Serbia (Grant No. 41010).

\section{References}

ANDREws, J.M. (2005): BSAC standardized disc susceptibility testing method (version 4). J. Antimicrob. Chemotherapy, 56, 60-76.

BAKоČEviĆ, I. (2011): White paper - Preservatives, introduction and classification. Food technology, http://www. tehnologijahrane.com/enciklopedija/konzervansi-uvod-i-podela. (Last accessed: 10 August 2017)

Bhasin, S. \& Modi, H.A. (2012): Optimization of fermentation medium for the production of glucose isomerase using Streptomyces sp. SB-P.1. Biotechnol. Res. Int., Article ID 874152, 10 pages.

BRADFORD, M.M. (1976): A rapid and sensitive method for the quantitation of microgram quantities of protein utilizing the principle of protein-dye binding. Anal. Biochem., 7, 248-254.

BRyAnt, A.M. (1965): The effect of nitrate on the in vitro fermentation of glucose by rumen liquor. J. Agric. Res., 8 , $118-125$.

Campos, C.A., Castro, M.P., Gliemmo, M.F. \& Schelegueda, L.I. (2011): Use of natural antimicrobials for the control of Listeria monocytogenes in foods. -in: MÉNDEZ-VILAS, A. (Ed.) Science against microbial pathogens: Communicating current research and technological advances. Formatex, Badajoz, Spain, pp. 1112-1123.

Cox, S.D., Mann, C.M., Markham, J.L., Gustafson, J.E., Warmington, J.R. \& Wyllie, S.G. (2001): Determining the antimicrobial actions of tree oil. Molecules, 6, 87-91. 
DenYer, S.P. \& Hugo, W.B. (1991): Biocide-induced damage to the bacterial cytoplasmic membrane. -in: DenYeR, S.P. \& Hugo, W.B. (Eds), Mechanisms of action of chemical biocides: their study and exploitation. Oxford, Blackwell Scientific Publications, England, pp. 171-187.

EkLund, T. (1985a): The effect of sorbic acid and esters of hydroxybenzoic acid on the proton motive force in Escherichia coli membrane vesicles. J. Gen. Microbiol., 131, 73-76.

EKLUND, T. (1985b): Inhibition of microbial growth at different $\mathrm{pH}$ levels by benzoic and propionic acids and esters of p-hydroxybenzoic acid. Int. J. Food. Microbiol., 2, 159-167.

Hasan, S., Ahmad A., Purwar, A., Khan, N., Kundan, R. \& Gupta, G. (2013): Production of extracellular enzymes in the entomopathogenic fungus Verticillium lecanii. Bioinformation, 9, 238-242.

Nikaido, H. \& VAaRA, T. (1985): Molecular basis of bacteria outer membrane permeability. Microbiol. Mol. Biol. R., $49,1-32$.

Oladapo, A.S., Akinyosoye, F.A. \& Abiodun, O.A. (2014): The inhibitory effect of different chemical food preservatives on the growth of selected food borne pathogenic bacteria. Afr. J. Microbiol. Res., 8, 1510-1515.

Piper, P., Ortiz, C.C., Kostas, H. \& Mehdi, M. (2001): Weak acid adaptation: the stress response that confers yeasts with resistance to organic acid food preservatives. Microbiology, 147, 2635-2642.

Rojo-Bezares, B., Saenz, Y., Zarazaga, M., Torres, C. \& Ruiz-Larres, F. (2007): Antimicrobial activity of nisin against Oenococcus oeni and other wine bacteria. Int. J. Food Microbiol., 116, 32-36.

Russell, A.D. \& Gould, G.W. (1988): Resistance of Enterobacteriaceae to preservatives and disinfectants. J. Appl. Microbiol., 65, 167-195.

Sarker, S.D., Nahar, L. \& Kumarasamy, Y. (2007): Microtitre plate-based antibacterial assay incorporating resazurin as an indicator of cell growth, and its application in the in vitro antibacterial screening of phytochemicals. Methods, 42, 321-324.

SIJM, D. (2001): Benzoates. OECD SIDS initial assessment report for 13th SIAM, 7-9 November, Bern, Netherlands, pp. $90-97$.

Stecchini, M.L., Torre, M. \& Polese, P. (2013): Survival strategies of Bacillus spores in food. Ind. J. Exp. Biol., 51, 905-909.

Vartiainen, J., Skytta, E., Enqvist, J. \& Ahvenainen, R. (2003): Properties of antimicrobial plastics containing traditional food preservatives. Packag. Technol. Sci., 16, 223-229.

Yaganza, E., Tweddell, R. \& Arul, J. (2009): Physicochemical basis for the inhibitory effects of organic and inorganic salts on the growth of Pectobacterium carotovorum subsp. carotovorum and Pectobacterium atrosepticum. Appl. Environ. Microb., 75, 1465-1469. 